\title{
Analyses of DNA content of living spermatozoa using flow cytometry techniques
}

\author{
D. W. Dresser ${ }^{1}$, C. J. Atkins ${ }^{1}$, A. Pinder ${ }^{2}$ and J. M. Morrell ${ }^{1, *}$ \\ ${ }^{1}$ National Institute for Medical Research, London NW7 1AA, UK; and ${ }^{2} A F R C$ Institute of Food \\ Research, Nonwich Laboratory, Colney Lane, Norwich NR4 7UA, UK
}

\begin{abstract}
A two-dimensional fluorescence analysis of spermatozoa stained with Hoechst 33342 was carried out using an Epics $\mathrm{V}$ flow cytometer. This analysis involved the measurement of fluorescence both in the conventional manner at $90^{\circ}$ and at a narrow forward angle (nominally $0^{\circ}$ ) to the direction of the interrogating laser beam. The arrangement provided an optical means of distinguishing the orientation of flattened particles passing through the interrogation system and enabled the verification and reinterpretation of one-dimensional $\left(90^{\circ}\right)$ fluorescence histogram data obtained previously. We now consider that the distinction between live and dead spermatozoa was over simplified and that the observed differences are due partly to an artefact of orientation. It has been confirmed that our earlier use of the low fluorescence peak, consisting of (bull, rabbit, sheep and pig) spermatozoa passing through the interrogation point obliquely to both detectors, as criterion for sorting $\mathrm{X}$ - from $\mathrm{Y}$-bearing spermatozoa, is more practical than the use of a very much smaller subpopulation, based on limits imposed by selecting only spermatozoa accurately orientated with their narrow edges to one detector, while measurement of total fluorescence (DNA content) was made simultaneously from a broad face by the other detector. We report marked differences in the rate of staining and in attainment of a staining equilibrium between spermatozoa from different animals or from different ejaculates collected on the same or different days from the same animal. This variation introduces an element of subjectivity into the use of flow cytometry for sorting X- and Y- spermatozoa. Spermatozoa from rabbits, mice and chickens were also examined using the two-dimensional system.
\end{abstract}

\section{Introduction}

Accurate estimates of the DNA content of fixed spermatozoa stained with fluorochromes such as ethidium bromide, mithramycin or acridine orange, using flow cytometric techniques, were first made over a decade ago using fixed spermatozoa (Van Dilla et al., 1977; Gledhill et al., 1979). Subsequently, less accurate but biologically more relevant measurements were made of viable motile spermatozoa stained with bis-benzimidazole dyes such as Hoechst 33258 and 33342 (H.33258; H.33342) (Keeler, ef al., 1983). Live spermatozoa stained with H.33342 were found to have a major low fluorescent peak with a narrowly separated bimodal distribution: the peak with the greater fluorescence was assumed to represent $X$-bearing spermatozoa, the lower peak Y-bearing spermatozoa. At that time one or two more highly fluorescent peaks were shown to be largely composed of moribund spermatozoa. Spermatozoa sorted by a flow cytometer-sorter into putatively $\mathrm{X}$ - and $\mathrm{Y}$ enriched populations were inseminated into suitably prepared females and gave rise to progeny (cattle and rabbits) which showed an overall statistically significant bias from the normal towards the expected sex ratio (Morrell et al., 1988). These

*Present address: Deutsches Primatenzentrum, Kellnerweg 4, D-37077, Göttingen, Germany.

Received 29 June 1992. early studies were extended and refined by Johnson et al. (1989) using a Coulter Epics V flow cytometer-sorter modified to measure fluorescence both orthogonally $\left(90^{\circ}\right)$ in the current standard manner for commercially available machines and at a narrow forward angle $\left(0^{\circ}\right)$ in the manner of earlier Ortho Cytofluorographs. On the basis of their studies, Johnson et al. (1989) concluded that they could distinguish the physical orientation of spermatozoa in the interrogated stream by using the $90^{\circ}$ detector to identify spermatozoa orientated with their narrow edges towards the $90^{\circ}$ detector and consequently their flat sides towards the $0^{\circ}$ detector, which was used to make accurate estimates of DNA content and thus to distinguish between $X$ and $Y$-bearing spermatozoa for sorting. Unfortunately, in their system, the population that meets the necessary criteria for analysis for sorting is small and since the total throughput is limited to 2500 cells s$^{-1}$ or less, the yield of successfully separated $X$-and $Y$-bearing spermatozoa is low. We demonstrate that the ability to detect spermatozoa accurately orientated with their narrow edges towards one or other detector probably depends on a lens-like effect of the head. Assuming that spermatozoa are randomly orientated around their long axes in the flow stream, those with their narrow edges precisely normal to a detector constitute a very small proportion of the total throughput: the majority are obliquely orientated. This oblique population is shown to be sufficiently homogeneous with 
Table 1. Dilution of ejaculates and staining of spermatozoa for flow cytometric analysis

\begin{tabular}{|c|c|c|c|}
\hline Animal & Diluent & Procedure & $\begin{array}{c}\text { Stain } \\
\left(\mu \mathrm{g} \mathrm{ml}^{-1}\right)\end{array}$ \\
\hline Bull & TALP & $\begin{array}{l}\text { Semen added to equal } \\
\text { volume warm diluent, } \\
\text { then } 200 \mu 1 \text { to } 10 \mathrm{ml} \\
\text { with stain }\end{array}$ & $\begin{array}{l}\text { H.33342 and } \\
\text { H.33258 }(1,2,4,8)\end{array}$ \\
\hline Rabbit & Citrate & Ejaculate diluted to $5 \mathrm{ml}$ & H.33342 (4) \\
\hline Mouse & $\begin{array}{l}\text { Citrate } \\
\quad \text { or } \mathrm{M} 2\end{array}$ & $\begin{array}{l}\text { Contents of two } \\
\text { epididymides to } 1 \mathrm{ml}\end{array}$ & H.33342 (2) \\
\hline Cock & Poultry & Semen diluted $\times 2$ at $20^{\circ}$ & H.33342 $(2,4,8)$ \\
\hline
\end{tabular}

Diluents: TALP: NaCl $5.4 \mathrm{~g} ; \mathrm{KCl} 0.23 \mathrm{~g} ; \mathrm{Na}_{2} \mathrm{HPO}_{4} 0.04 \mathrm{~g} ; \mathrm{MgCl}_{2} .6 \mathrm{H}_{2} \mathrm{O} 0.31 \mathrm{~g}$; $\mathrm{NaHCO}_{3} 2.1 \mathrm{~g}$; HEPES $2.38 \mathrm{~g}$; Phenol Red $0.01 \mathrm{~g}$; Penicillin $0.06 \mathrm{~g}$; Streptomycin $0.1 \mathrm{~g}$; sodium pyruvate $0.11 \mathrm{~g}$; sodium lactate $(70 \%) 3.68 \mathrm{ml}$; BSA Fraction V $6.0 \mathrm{~g}$; double distilled water to $\mathrm{Il}-291 \mathrm{mOsm}$; $\mathrm{pH} 7.3 \mathrm{in} 5 \% \mathrm{CO}_{2}$ filter through $0.22 \mu \mathrm{m}$ millipore.

Citrate: $2.9 \% \mathrm{w} / \mathrm{v}$ tri-sodium citrate, $\mathrm{pH}$ adjusted to 6.9 with $10 \% \mathrm{w} / \mathrm{v}$ citric acid.

$\mathrm{M} 2: \mathrm{NaCl} 5.53 \mathrm{~g} ; \mathrm{KCl} 0.36 \mathrm{~g} ; \mathrm{CaCl}_{2}, 2 \mathrm{H}_{2} \mathrm{O} 0.25 \mathrm{~g} ; \mathrm{KH}_{2} \mathrm{PO}_{4} 0.16 \mathrm{~g} ; \mathrm{MgSO}_{4}$. $7 \mathrm{H}_{2} \mathrm{O} 0.29 \mathrm{~g} ; \mathrm{NaHCO}_{3} 0.35 \mathrm{~g}$; HEPES $4.97 \mathrm{~g}$; Phenol Red $0.01 \mathrm{~g}$; Penicillin $0.06 \mathrm{~g}$; Streptomycin $0.05 \mathrm{~g}$; sodium pyruvate $0.04 \mathrm{~g}$; sodium lactate $(70 \%)$ $3.68 \mathrm{ml}$; glucose $1.0 \mathrm{~g}$; BSA Fraction V $4.0 \mathrm{~g}$; double distilled water to II 286 mOsm; pH 7.3 filter through $0.22 \mu \mathrm{m}$ millipore (Hogan et al., 1986).

Poultry: $\mathrm{NaCl} 8.0 \mathrm{~g}$; TES (Sigma) $13.74 \mathrm{~g}$; glucose $6.0 \mathrm{~g}$; Penicillin $0.06 \mathrm{~g}$; Streptomycin $0.05 \mathrm{~g}$; double distilled water to $1 \mathrm{l}-\mathrm{pH} 7.4$ with $2-3 \mathrm{ml} 1 \mathrm{~mol}$ $\mathrm{NaOH}^{-1}$.

respect to fluorescence emission, to be used for the separation of $X$ - and $Y$-spermatozoa.

The Coulter Epics V flow cytometer-sorter was altered so that both $0^{\circ}$ and $90^{\circ}$ fluorescence could be measured simultaneously and the modified machine was used to make extensive two-dimensional analyses of living bull, rabbit, dog, mouse and cock spermatozoa.

\section{Materials and Methods}

\section{Flow cytometry}

A Becton-Dickinson FACStar plus and a modified Coulter Epics $V$ were used in this study. Unless stated otherwise, $50 \mathrm{~mW}$ of $350-356 \mathrm{~nm}$ laser light (UV) was provided by Innova UV enhanced 90-5 lasers. Preliminary experiments showed that outputs in the range $15-100 \mathrm{~mW}$ were equally sensitive and gave equivalent results when the photomultiplier tube (PMT) amplification gain was adjusted appropriately.

Our Coulter Epics V flow cytometer was modified as follows. An aperture was cut in the endplate of the instrument (approximately $30 \mathrm{~mm}$ by $80 \mathrm{~mm}$ ) immediately behind the forward scatter detector and an optical rail fitted to extend the optical path length by $256 \mathrm{~mm}$. A special mount was constructed to enable one of the standard Coulter PMTs to be fitted on to the optical rail, together with an adjustable image aperture and a relay lens (all components from Micro-Controle, Newbury). The forward scatter detector diode could then be unscrewed and the 'fluorescence $3^{\prime}$ PMT moved from its normal position to the new forward angle position, to measure two-dimensional fluorescence at $0^{\circ}$ and $90^{\circ}$, respectively. Both detection channels were fitted with a $408 \mathrm{~nm}$ long-pass optical filter.

Stovel et al. (1978) showed that flattened particles could be physically orientated in the hydrodynamically focussed stream passing through the interrogation point of a flow cytometer, by shaping the sample injection needle, such that the sample stream, at the centre of the sheath stream, formed a ribbon rather than the more usual cylinder. For a limited number of experiments, described in the results section, a similar modification was made to the Coulter Epics V by grinding one of the standard sample injection needles to a $20^{\circ}$ (from the long axis of the needle) chisel shape. The effectiveness of this modification was verified by a standard forward angle scatter analysis of glutaraldehyde fixed turkey erythrocytes as described by Keeler et al. (1983). The injection needle could be rotated so that the ribbon was at any desired position between $0^{\circ}$ and $90^{\circ}$ with respect to the laser beam. Johnson et al. (1989) used a similarly modified sample injection needle in some of their experiments.

Data for each analysis of 20000 spermatozoa was stored on the computer system (in list mode) with relatively wide limits for each parameter. This enables more stringent limits to parameters to be set to select subpopulations for detailed computer-based analysis.

\section{Spermatozoa}

Semen was collected from bulls, rabbits and cocks using methods of Napier (1963) and Moss et al. (1979). Spermatozoa were stained with Hoechst 33342 (H.33342; H.33258 was used in a few experiments) by diluting the spermatozoa suspensions in stain at various concentrations (see Table 1). Mouse epididymal spermatozoa were obtained by mincing the epididymides in $1 \mathrm{ml}$ medium and filtering the resulting suspension through $45 \mu \mathrm{m}$ nylon mesh to remove extraneous material.

\section{Results}

There can be considerable variation in the fluorescence of H.33342 and H.33258 stained spermatozoa from an individual bull. For example, the result of staining spermatozoa from two ejaculates obtained about $30 \mathrm{~min}$ apart, with two different concentrations of H.33342 for either $3 \mathrm{~h}$ or $6 \mathrm{~h}$ in TALP (see Table 1) are shown (Fig. 1). Almost identical results were obtained for spermatozoa stained with H.33258 when these were suspended in TALP: H33258 is unsuitable for use with milk or egg yolk based diluents (J. Morreu, unpublished observations). Greater differences can be seen between spermatozoa from the same bull collected on different days. There is a clear suggestion from the data presented (Fig. 1) that the staining level also depends on the time and concentration of dye in the medium. These experiments were carried out at $20-23^{\circ}$ : other experiments with rabbit and bull spermatozoa have shown that staining at $37^{\circ}$ achieves an equilibrium in a shorter time (approximately 40-60 min). In many early experiments, where measurements were made of the fluorescence profiles of duplicate samples from the same ejaculate stained in the same manner, the fluorescence profiles were found to be indistinguishable. We therefore concluded that the observed differences were not due to technical artefacts. 
(a)

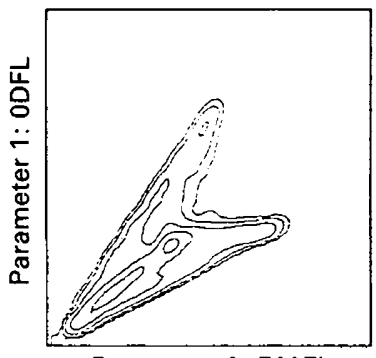

Parameter 3: P90FL (b)

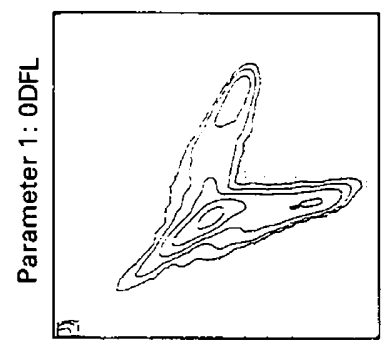

Parameter 3: P9OFL (c)

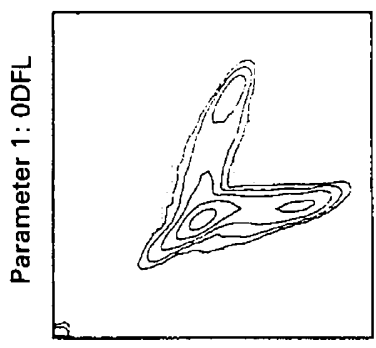

Parameter 3: P90FL (d)

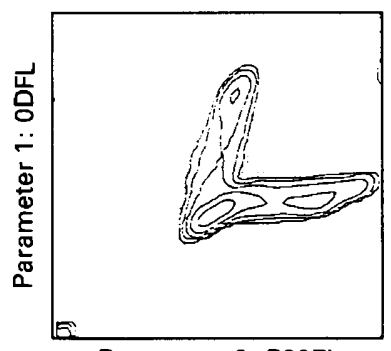

Parameter 3: P90FL (e)

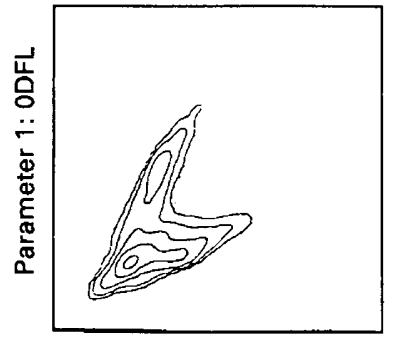

Parameter 3: P90FL (f)

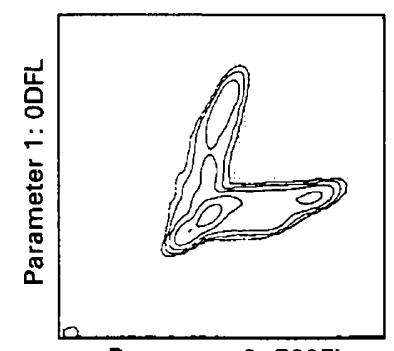

Parameter 3: P90FL (g)

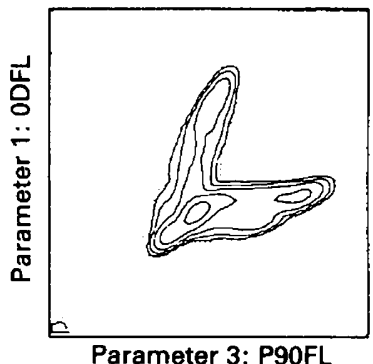

Parameter 3: P90FL (h)

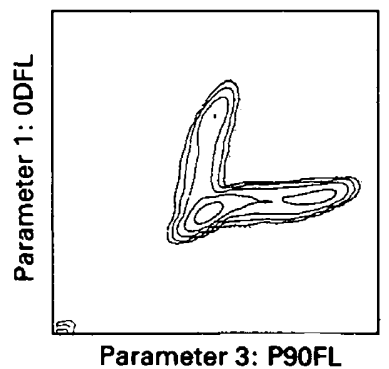

Fig. 1. Spermatozoa from Friesian bull (Komeet) stained with Hoechst 33342. The effect of ejaculate number, concentration of the fluorochrome and time of staining as illustrated in two-dimensional plots of fluorescence measured at a narrow forward angle to the interrogating laser beam $\left(0^{\circ}, \mathrm{ODFL}\right)$ and at right angles to the beam $\left(90^{\circ}, \mathrm{P} 9 \mathrm{OFL}\right)$. The contour lines join levels with equal numbers of spermatozoa for each combination of values for ODFL and P9OFL. (a), (e), First ejaculate, $2 \mu \mathrm{g}$ stain; (b), (f), first ejaculate, $8 \mu \mathrm{g}$ stain; (c), (g), second ejaculate, $2 \mu \mathrm{g}$ stain; (d), (h), second ejaculate, $8 \mu \mathrm{g}$ stain. (a, b, c, d) stained for $3 \mathrm{~h}$; (e, f, $g$, h) stained for $6 \mathrm{~h}$.

(a)

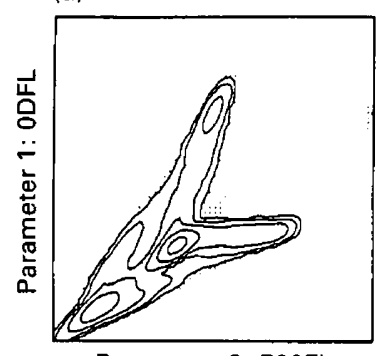

Parameter 3: P90FL (b)

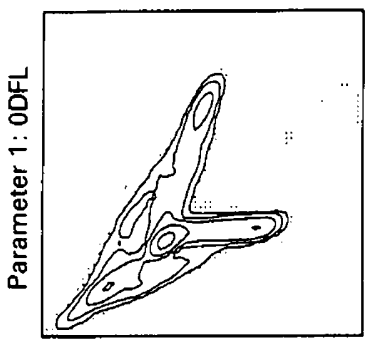

Parameter 3: P90FL (c)

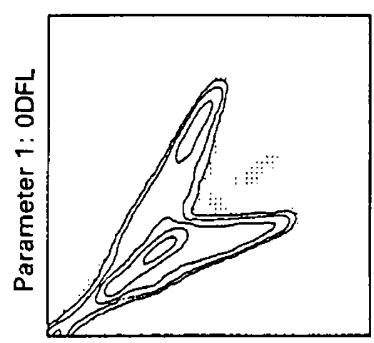

Parameter 3: P90FL

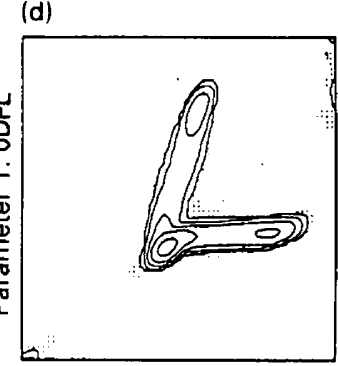

Parameter 3: P90FL (e)

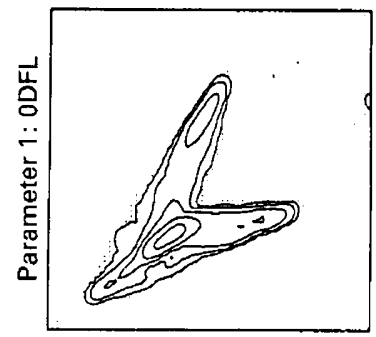

Parameter 3: P90FL (f)

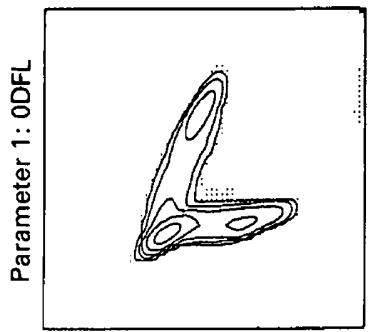

Parameter 3: P9OFL

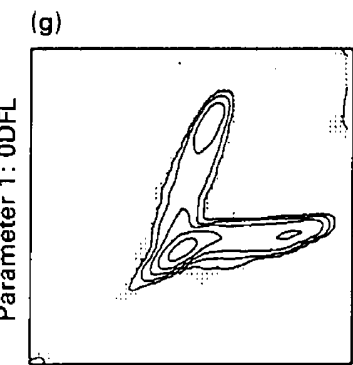

Parameter 3: P90FL

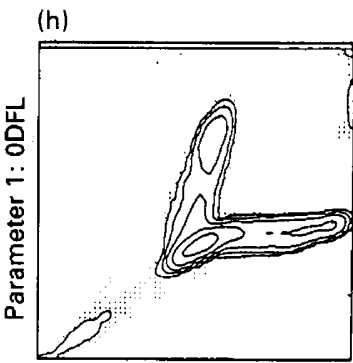

Parameter 3: P90FL

Fig. 2. Bull spermatozoa analysed on the same day as those analysed in Fig. 1. Heterogeneity between spermatozoa from the first ejaculates of two Friesian bulls, Claret and Sheik Bell. Spermatozoa stained with a range of four concentrations of $\mathrm{H} .33342 \mathrm{for} 3 \mathrm{~h}$. Two-dimensional analysis similar to that described for Fig. 1. (a, b, c, d) spermatozoa of Claret stained with $1,2,4$ and $8 \mu \mathrm{g}$ fluorochrome, respectively; $(e, f, g, h)$ spermatozoa from Sheik Bell also stained with $1,2,4$ and $8 \mu \mathrm{g}$, respectively. 


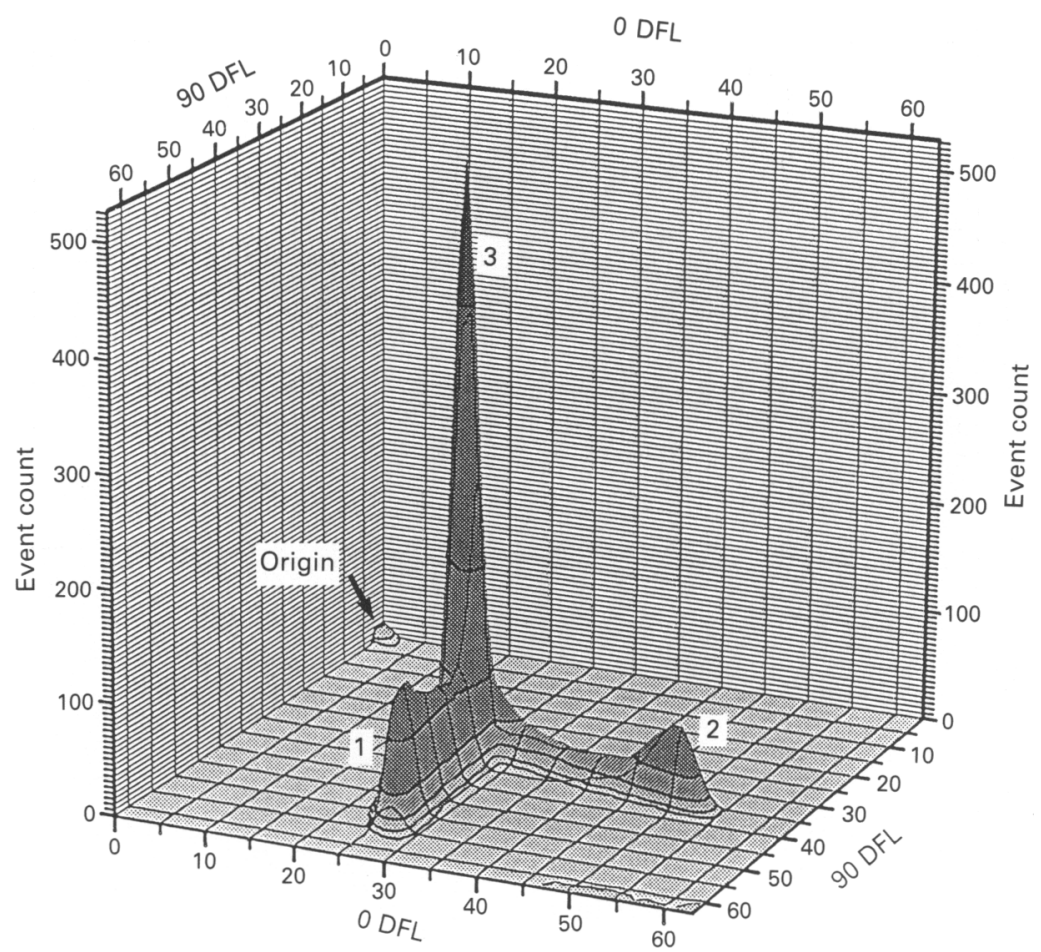

Fig. 3. A three-dimensional plot using linear parameters to emphasize the relative sizes and distribution of the subpopulations. Same data as in Fig. 2(d). Fluorescence measured at a narrow forward angle to the interrogating laser beam $\left(0^{\circ}, 0 \mathrm{DFL}\right)$ and at right angles to the beam $\left(90^{\circ}, 90 \mathrm{DFL}\right)$. 'Event count' is the number of spermatozoa in each category. Peak 1 represents spermatozoa with a narrow edge precisely orientated towards the $90^{\circ}$ detector and peak 2 those with a narrow edge towards the $0^{\circ}$ detector. Peak 3, consisting of about $50 \%$ of total throughput, represents spermatozoa lying obliquely to both detectors.

An experiment using spermatozoa from two bulls in which a range of concentrations of $\mathrm{H} .33342$ was used to stain spermatozoa in TALP for $3 \mathrm{~h}$ are shown (Fig. 2). Although dye concentration above $8 \mu \mathrm{g}$ was shown not to change the pattern further for any bull (staining equilibrium), the spermatozoa of some bulls (e.g. Sheik Bell) appear to reach a staining equilibrium at lower concentrations. The plots presented (Fig. 2) give a good qualitative representation of changes in staining but they fail to give an accurate impression of the relative numbers of spermatozoa in each area of the plot. This omission is rectified in a three-dimensional plot (Fig. 3), with linear axes, of the equilibrium data for Claret (Fig. 2(d)). It can be seen that the major peak (containing about $50 \%$ of spermatozoa passing through the interrogation system), represents spermatozoa that have low $0^{\circ}$ and $90^{\circ}$ fluorescence. Experiments (summarized in Fig. 4 and Table 2) suggest that this population represents spermatozoa that are oblique to both fluorescence detectors.

A photomicrograph of living (motile) bull spermatozoa in TALP at $20^{\circ}$, stained with H.33342, illuminated by UV and fluorescing in the visible spectrum is shown (Fig. 5). Spermatozoa seen edge-on were considerably brighter than spermatozoa seen obliquely or face-on.

Stovel et al. (1978) showed that flattened particles could be orientated in a specific manner in the inner part of the interrogation stream of a flow cytometer, by using a chisel-shaped sample injection needle. The technique is successful providing the flow rate is sufficiently low: in this study a rate of 400 cells $\mathrm{s}^{-1}$ or less was used. The spermatozoa of many mammals are flattened and consequently can give artefactual fluorescence distributions if interrogated in random orientation in a cylindrically shaped inner stream. The technique of Stovel $e t$ al. was used to investigate whether $0^{\circ}$ versus $90^{\circ}$ fluorescence can determine the orientation of spermatozoa passing through the flow cytometer. Two-dimensional plots of spermatozoa from Catlane Claret stained with $8 \mu \mathrm{g} \mathrm{H} .33342$ for $3 \mathrm{~h}$ at $22^{\circ}$ are shown (Fig. 4): from the previous data it was assumed that this was equilibrium staining. The standard distribution for the circular injection needle is shown in plot (a), which has been arbitrarily divided into four quadrants. Quadrant I represents spermatozoa with their narrow edges towards the $0^{\circ}$ detector, quadrant IV spermatozoa with their narrow edges towards the $90^{\circ}$ detector and spermatozoa with their broad faces lying obliquely to both detectors are in quadrant III. The data in Fig. 4 are summarized (Table 2) as the ratio of the proportion of spermatozoa in quadrants I:III and IV:III. An increase in the ratio of I:III indicates an increase in the number of spermatozoa that are orientated with their edges towards the $0^{\circ}$ detector and vice versa for IV:III. Plots (b), (c) and (d) were obtained using an orientating nozzle with a chisel-shaped injection needle. This needle was orientated at $90^{\circ}, 0^{\circ}$ or $45^{\circ}$, respectively, to the laser beam. It can be seen that there is a significant bias in the fluorescence ratios towards that expected from the physical 
(a)

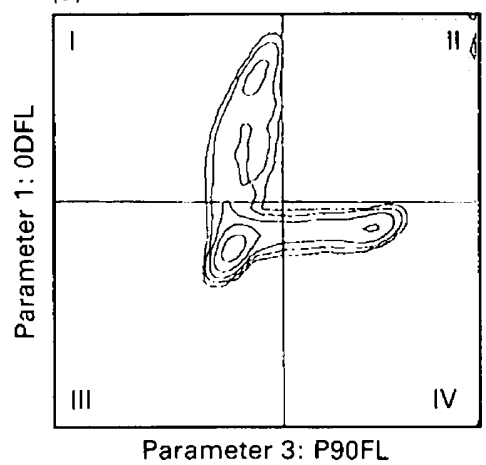

(c)

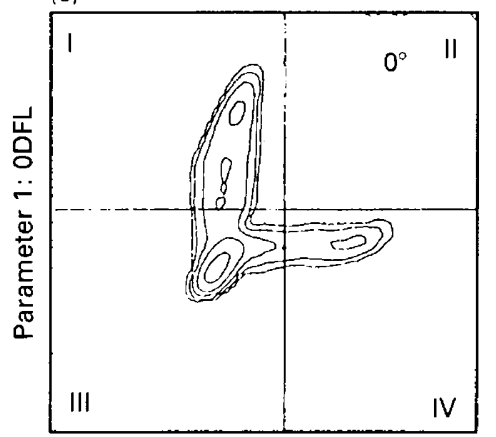

Parameter 3: P9OFL (b)

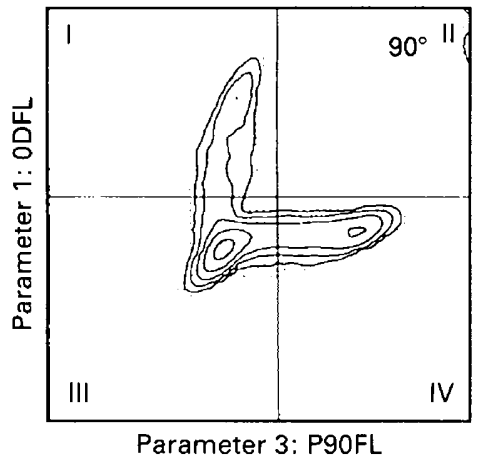

(d)

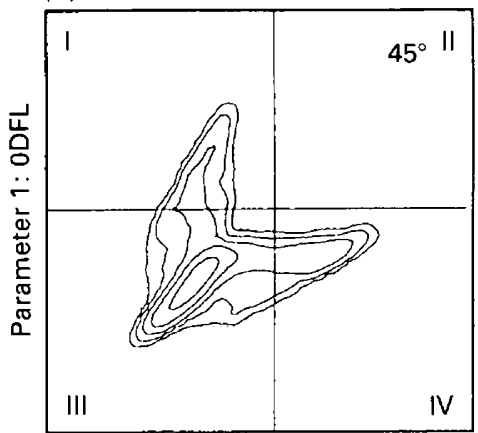

Parameter 3: P90FL

Fig. 4. The use of an orientating nozzle set at three different positions to verify the ability of the optical system to detect the orientation of flattened particles (spermatozoa) in the interrogated stream. A normal bifurcated pattern obtained using an injection nozzle with a circular orifice is shown in the top left in plot (a): this and the other plots are divided into four quadrants for convenience of further analysis (summarized in Table 2). Sector (b) represents the sample ribbon edge-on to the $90^{\circ}$ detector, (c) edge-on to the $0^{\circ}$ detector and (d) oblique to both detectors. Spermatozoa from Friesian bull Catlane Claret.

Table 2. Verification that fluorescence measured simultaneously at $0^{\circ}$ and $90^{\circ}$ can distinguish the orientation of flattened spermatozoa in the interrogation stream of a flow cytometer

\begin{tabular}{|c|c|c|c|c|}
\hline \multirow[b]{2}{*}{$\begin{array}{l}\text { Ratio of } \\
\text { quadrants: }\end{array}$} & \multicolumn{4}{|c|}{$\begin{array}{l}\text { Mean ratio ( } \pm \text { SEM) } \\
\text { Injection nozzle: }\end{array}$} \\
\hline & $\begin{array}{c}\text { Circular } \\
\text { (a) }\end{array}$ & $\begin{array}{l}90^{\circ} \\
\text { (b) }\end{array}$ & $\begin{array}{l}0^{\circ} \\
\text { (c) }\end{array}$ & $\begin{array}{l}45^{\circ} \\
(\mathrm{d})\end{array}$ \\
\hline I:III & $\begin{array}{l}0.38 \\
(0.005)\end{array}$ & $\begin{array}{l}0.19 \\
(0.006)\end{array}$ & $\begin{array}{c}0.53 \\
(0.04)\end{array}$ & $\begin{array}{c}0.12 \\
(0.003)\end{array}$ \\
\hline IV:III & $\begin{array}{c}0.44 \\
(0.01)\end{array}$ & $\begin{array}{c}0.50 \\
(0.02)\end{array}$ & $\begin{array}{c}0.19 \\
(0.02)\end{array}$ & $\begin{array}{c}0.16 \\
(0.007)\end{array}$ \\
\hline
\end{tabular}

$90^{\circ}$ and $0^{\circ}$ indicates that the ribbon-shaped sample stream at the centre of the sheath stream, which is generated by the chisel-shaped injection nozzle, is orientated with its narrow edge towards these respective detectors. $45^{\circ}$ indicates that the ribbon is at $45^{\circ}$ to both detectors. (a)-(d) refer to the four sectors of Fig. 4. Analyses carried out at an average throughput of $<400$ spermatozoa $\mathrm{s}^{-1}$. Mean ratio ( $\mathrm{SEM}$ ) of results from the analysis of spermatozoa of four bulls; two Holsteins, one Simintal and I Friesian whose spermatozoa were used for the analysis illustrated in Fig. 4.

orientation of the spermatozoa dictated by the orientating nozzle.

In the two-dimensional contour plot (Fig. 6) a window was drawn around values for spermatozoa with their narrow edges

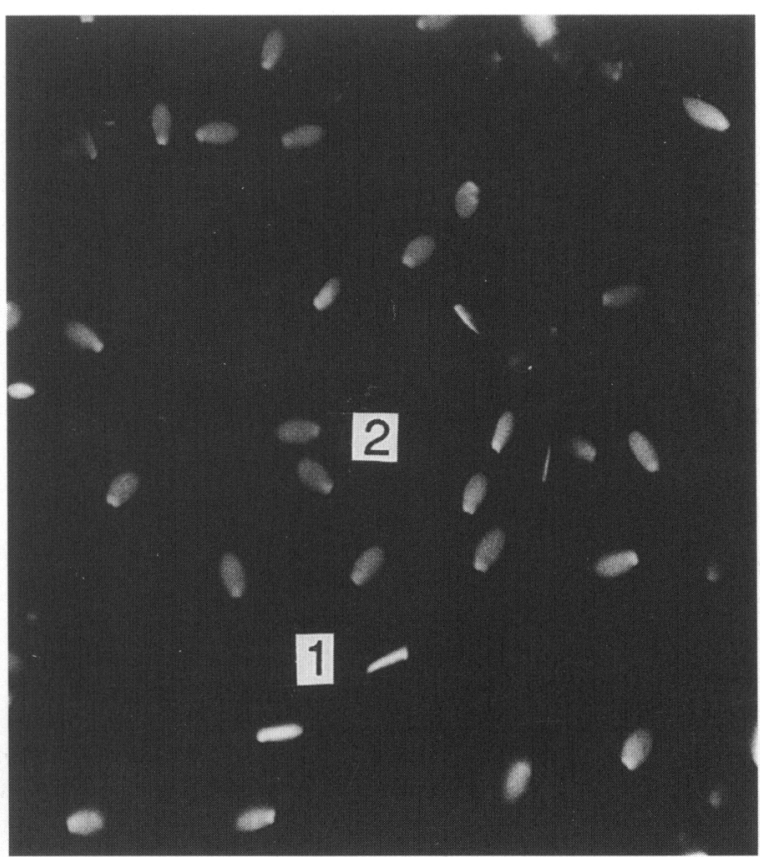

Fig. 5. Photomicrograph of living bull spermatozoa stained with $8 \mu \mathrm{g}$ H.33342 for $4 \mathrm{~h}$ (TALP). Dark ground UV illumination. Bright edge views (1) contrast with the less bright fluorescent face (about $9.0 \times 4.5 \mu \mathrm{m})(2)$.

Downloaded from Bioscientifica.com at 04/26/2023 03:07:28AM 
(b)

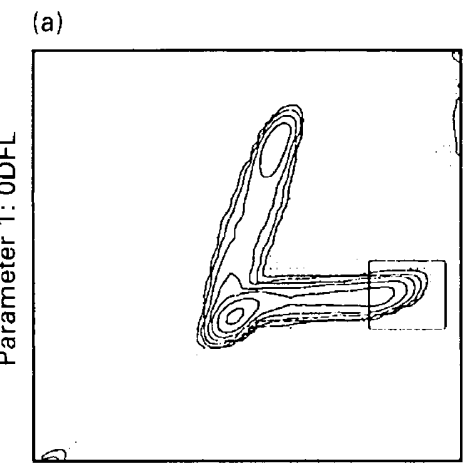

Parameter 3: P90FL

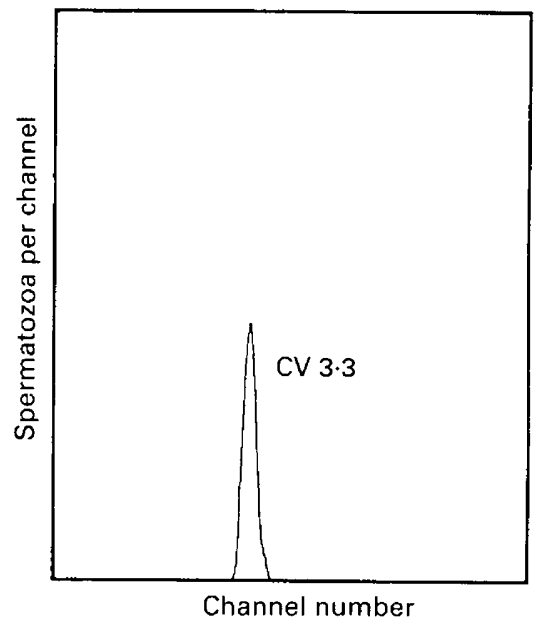

(d)

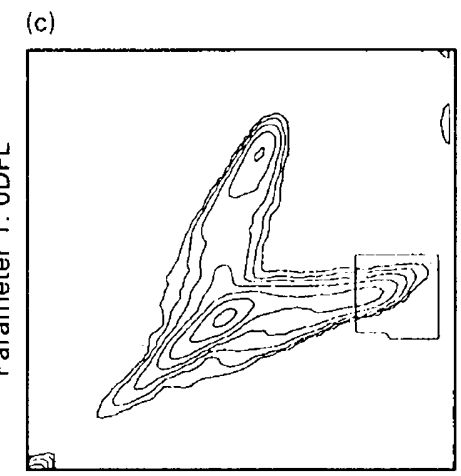

Parameter 3: P90FL

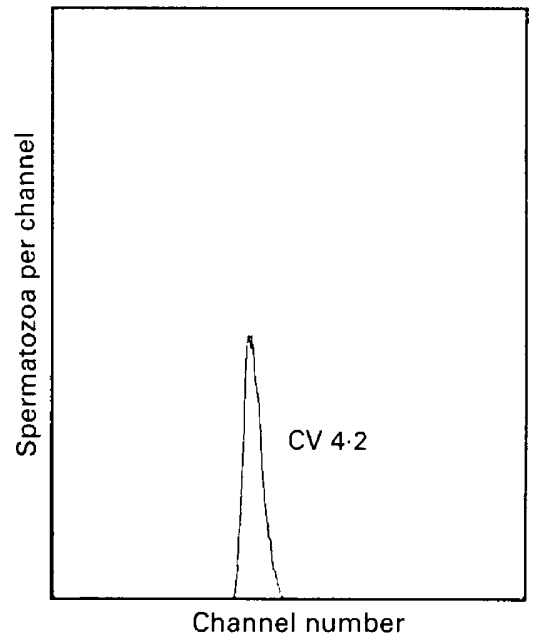

Fig. 6. Spermatozoa from the first ejaculates of two bulls (Claret and Komeet). Data stored as a list mode file for further analysis consisting of setting a window, as indicated on the two-dimensional plot and then plotting a one-dimensional histogram of the selected subpopulation. The coefficients of variation (CV) are indicated on each histogram. The CVs of five other similar analyses of spermatozoa from Friesian and Holstein bulls fell within the limits indicated in this figure. In no case was any bimodality observed in this population (peak $I$ in Fig. 3) that might represent the difference between $X$-and $Y$-bearing spermatozoa. (a,b) Spermatozoa from Claret stained with $8 \mu \mathrm{g} \mathrm{H.33342}$ for $3 \mathrm{~h}$. The two-dimensional plot represents a total throughput of 20000 spermatozoa, with 1845 spermatozoa in the windowed area represented as a one-dimensional histogram in (b). (c, d) are for Komeet, in a similar analysis to that shown in $(a, b)$; there were 1649 spermatozoa in the windowed area. In the histograms $(b, d)$ 'spermatozoa per channel' represents the number of spermatozoa in each channel, with increasing 'channel number' being directly related to increasing levels of fluorescence.

towards the $90^{\circ}$ detector, in a manner similar to that used by Johnson et al. (1989). The population within this window is reanalysed as a single parameter $\left(0^{\circ}\right)$ histogram. The proportion of the total throughput lying in the window is between 8.2 and $9.3 \%$; the corresponding range of coefficients of variation $(\mathrm{CV})$ for the seven animals analysed was from 4.2 to 3.3. A similar window drawn round the major peak of spermatozoa lying obliquely to both detectors, included about $50 \%$ of the total throughput. In this subpopulation of obliquely orientated spermatozoa there was evidence for an overlapping bimodal distribution with overall $\mathrm{CVs}$ in the range 5.0-6.0: this can be seen in histograms for both the $0^{\circ}$ and $90^{\circ}$ parameters in the example illustrated (Fig. 7). The bimodal peaks of fluorescence intensity (DNA content) were between 3 and $5 \%$ apart in the eight samples (from seven bulls) that were analysed.

Spermatozoa of other species were examined using the modified flow cytometer and examples of these analyses are presented (Fig. 8). It can be seen that the flattened or asymmetrical 
(a)

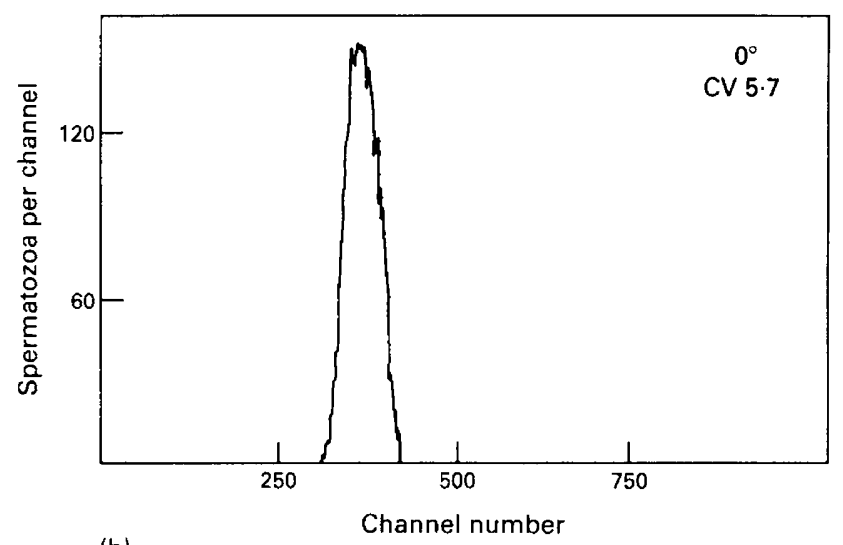

(b)

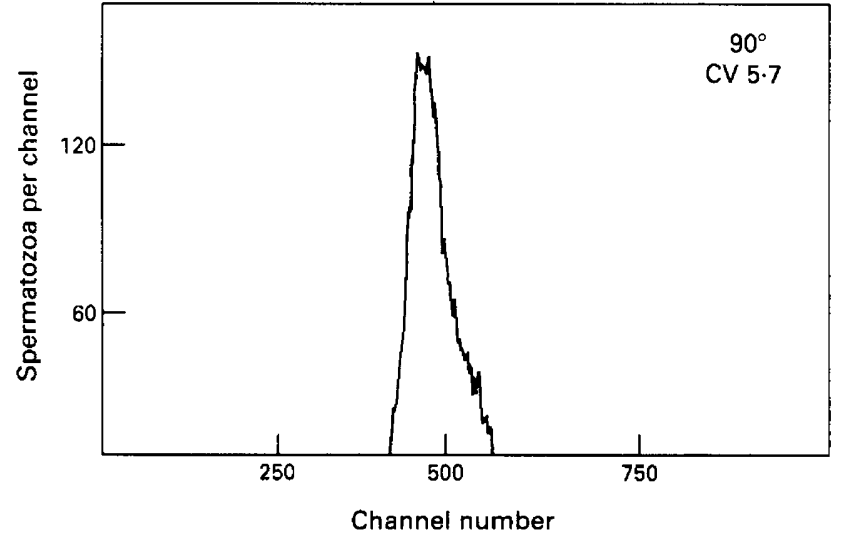

Fig. 7. Primary population of spermatozoa from Claret (as in Fig. 6). Window drawn round the major peak (peak 3 in Fig. 3) of spermatozoa passing obliquely to both detectors. Histograms drawn for both (a) $0^{\circ}$ and (b) $90^{\circ}$ parameters. The CVs are indicated on the figure. The narrowly defined bimodality seen in these histograms was also seen for spermatozoa of the six other bulls analysed.

spermatozoa of rabbits and mice both show bifurcated distributions in the two-dimensional plots. Cock spermatozoa (and dog spermatozoa - results not included) are more or less symmetrical round their long axes and were the only spermatozoa examined that did not have a distinctly bifurcated distribution.

\section{Discussion}

A two-dimensional flow cytometric analysis of spermatozoa stained vitally for DNA content was described. For this purpose a Coulter Epics $V$ flow cytometer was modified to allow detection of $0^{\circ}$ and $90^{\circ}$ fluorescence. This two-dimensional analysis technique was first implemented on Ortho Cytofluorograph machines and was used by Johnson et al. (1989) for the analysis and sorting of mammalian spermatozoa. Our implementation of the technology has enabled us to make detailed analyses of spermatozoa and confirm some of our earlier conclusions (Keeler et al., 1983; Morrell et al., 1988). It is clear that there is a considerable and largely uncontrollable variation in the results of analyses of bull spermatozoa which stem from factors both within and between individuals of the same breed. Dilution media (semen extenders), concentration of stain, temperature and duration of staining are all shown to be controllable factors that influence the vital staining pattern of spermatozoa as revealed by the two-dimensional analysis technique. The methodology has enabled us to distinguish the orientation of radially asymmetrical (flattened) particles such as bull and rabbit spermatozoa, by optical means. This conclusion was confirmed by the results of experiments using a chisel-shaped injection needle which physically orientates flattened particles before passage through the interrogating laser beam (Stovel et al., 1978).

Spermatozoa in the sample stream of a conventional flow cytometer with a circular blunt-ended sample injection nozzle will be randomly orientated round their long axes when they pass through the interrogation point: the long axis will be orientated in the direction of flow (Van Dilla et al., 1977). Johnson et al. (1989) used a two-dimensional detection system on a modified Coulter Epics V flow cytometer to identify accurately those rabbit spermatozoa that were orientated with their narrow edges towards the $90^{\circ}$ detector. Using the $0^{\circ}$ detector, they simultaneously measured the fluorescence of the spermatozoa in the defined subpopulation and could demonstrate a clear-cut bimodal distribution which was used as the basis for flow sorting. The spermatozoa in the two peaks were shown to represent $X$ - and $Y$ - spermatozoa in insemination experiments. However, we could not demonstrate a similar bimodality in the equivalent subpopulation of bull spermatozoa. A general disadvantage of the windowing technique of Johnson et al. (1989) is that the subpopulation so defined is $<10 \%$ of a total throughput restricted to below 2500 spermatozoa $\mathrm{s}^{-1}$, if optimum resolution is to be maintained. We showed in the study reported here that, at least for bull spermatozoa, the low fluorescence subpopulation (about $50 \%$ throughput) comprising spermatozoa passing through the interrogation point obliquely to both detectors, gives at least as good if not better potential resolution of $X$ - and $Y$ - spermatozoa than the smaller $(<10 \%)$ subpopulation defined by Johnson's method. In earlier work (Keeler et al., 1983; Morrell et al., 1988, 1989), on the basis of one-dimensional histogram data, this subpopulation was selected for sorting $X$ - and $Y$-spermatozoa. The spermatozoa in this subpopulation pass through the interrogation point with their heads oblique to both detectors: the subpopulation comprises about $50 \%$ of total throughput and thus results in higher yields in flow sorting than the alternative more narrowly defined subpopulation. It is relevant to note that our earlier work was carried out using a FACS II machine and that analysis of spermatozoa is notoriously sensitive to unexplained differences between flow cytometers (Shapiro, 1988). In several instances in the experiments reported here, small differences were observed in one-dimensional data obtained from the same samples, between the FACStar plus and the Epics V machines (data not included).

It seems reasonable to assume that when using a circular (blunt-ended) sample injection nozzle, the spermatozoa will be orientated at random around their long axes when passing through the interrogation point. Nevertheless the distribution of fluorescence emission values indicates that these values do not show a smooth continuous relationship with the orientation of the interrogated spermatozoa. A lens-like effect of the spermatozoa head seems to concentrate a large proportion of the emitted light to a narrow band normal to the narrow edge. When seen in the UV microscope, living spermatozoa exhibit 
(a)

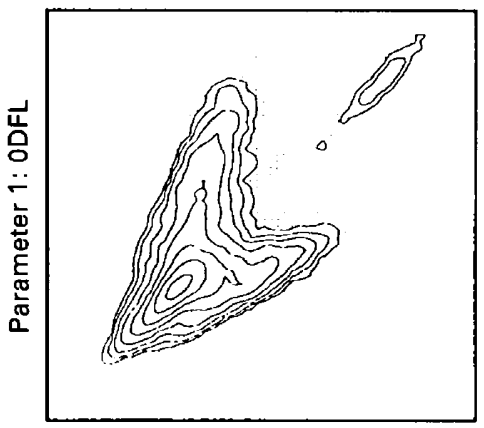

Parameter 3: P90FL

(c)

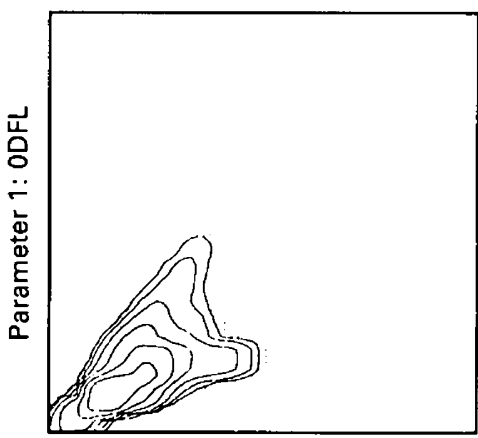

Parameter 3: P9OFL (b)

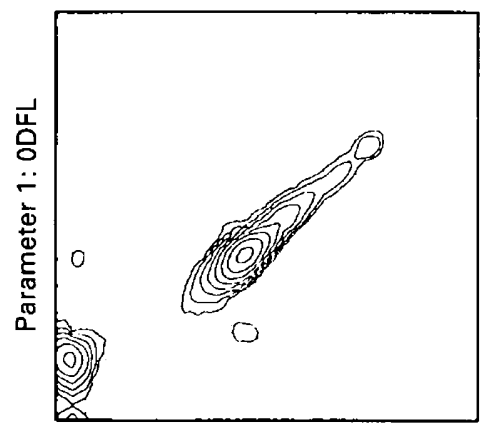

Parameter 3: P90FL

(d)

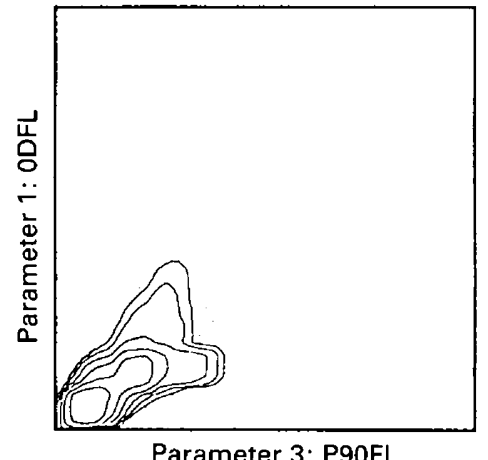

Fig. 8. Analyses of spermatozoa from (a) mouse, (b) cock, (c) rabbit and (d) rabbit, using optical detection of the orientation of flattened or non-axially symmetrical spermatozoa (rabbit and mouse, respectively). Cock spermatozoa are more or less cylindrical in shape and consequently have axial symmetry. It is important to note that all intact spermatozoa pass through the interrogation system with their long axes in the direction of flow (Van Dilla et al., 1977). It can be seen that only cock spermatozoa (and dog spermatozoa - results not included) do not have a bifurcated distribution in this analysis. The distinct population close to the origin in the illustration of the cock analysis, coincides with the position of unstained spermatozoa: there were some apparently unstained weakly autofluorescent spermatozoa in all six populations that were analysed.

a marked 'lighthouse effect', flashing brightly when viewed exactly edge-on as they rotate in the field of view. The concentration of the majority of the spermatozoa in the low fluorescence subpopulation indicates that there is a wide range of positions between the edge-on extremes, which do not affect the emitted light. This seems to be another argument in favour of using the subpopulation orientated obliquely to both detectors, for the measurement of small differences in DNA content in living spermatozoa.

Various factors can influence the physiology of sperm membranes in ways that may influence vital staining by bisbenzimidazole and other fluorochromes. For example, Evenson (1989) showed a progressive decrease in stainability by 7-diethyl-amino-3-(4'-maleimidylphenyl)-4-methylcoumarin of spermatozoa obtained respectively from the caput, corpus, and cauda epididymis of rats and mice. The time taken to reach a staining equilibrium is inversely related to temperature, suggesting dependence on a metabolic process. It is therefore interesting to note that Marengo and Amann (1990) described marked changes in the mitochondria of spermatozoa obtained from different regions of the epididymis. It seems likely that factors affecting male physiology may have a profound effect on the quality of ejaculated spermatozoa for flow cytometry analysis.

A reappraisal of our earlier study (Keeler et al., 1983) can now be made and it can be concluded that the original choice of the lower intensity peak was appropriate for sorting $X$ - and $\mathrm{Y}$-bearing bull spermatozoa at relatively high rates and that the previous interpretation of the double bimodal peaks seen in one-dimensional histograms of fluorescence may not represent a clear distinction between live and dead spermatozoa for all flow cytometers due perhaps to differences in design of injection nozzle and flow chamber. The previous use of a different machine (FACS II) may have contributed to our ability to distinguish live from dead spermatozoa using a one-dimensional system (Keeler et al., 1983; Morrell et al., 1988). The current use of a two-dimensional fluorescence system has made an important contribution towards our ability to reach this conclusion. Finally, in addition to demonstrating differences between individual animals, we have shown clearly that there can be considerable differences in fluorescence profile between stained spermatozoa from different ejaculates of the same 
animal. The idiosyncratic nature of the fluorescence profiles means that populations suitable for the separation of $X$ - and Y-bearing spermatozoa must be selected by flow cytometry analysis on a day-to-day basis.

We thank INNW fund for support, M. Philpot (Avoncraft Cattle Breeders, Bromsgrove, Birmingham) for his cooperation, N. Bumstead (Houghton Poultry Research Centre, Huntingdon) for providing cock spermatozoa from his experimental flock and R. J. Green (NIMR Computing Laboratory) for writing FACSPLOT, the flow cytometry data analysis package running under VAX VMS with GKS (requires $6.3 \mathrm{Mb}$ memory and VT340 or equivalent terminal).

\section{References}

Evenson JM (1989) Flowcytometric evaluation of male germ cells. In Flow Cytometry: Adoanced Research and Clinical Applications Vol. 1, p 225 Ed. A Yen. CRC Press, Florida

Gledhill BL, Lake S and Dean PN (1979) Flow cytometry and sorting of sperm and other male germ cells. In Flow Cytometry and Sorting PP 471-485 Eds ME Melamed, PF Mullaney and ML Mendelsohn. John Wiley, New York

Hogan B, Costantini F and Lacy E (1986) Manipulating the Mouse Embryo: a Laboratory Manual. Cold Spring Harbor Laboratory
Johnson LA and Pinkel D (1986) Modification of a laser-based flow cytometer for high-resolution DNA analysis of mammalian spermatozoa Cytometry 7 268-273

Johnson LA, Flook JP and Hawk HW (1989) Sex preselection in rabbits: live births from $X$ and $Y$ sperm separated by DNA and cell sorting Biology of Reproduction 41 199-203

Keeler KL, Mackenzie NM and Dresser DW (1983) Flow microfluorometric analysis of living spermatozoa stained with Hoechst 33342 Journal of Reproduction and Fertility 68 205-212

Marengo SR and Amann RP (1990) Morphological features of principal cells in the ovine epididymis: a quantitative study Biology of Reproduction $\mathbf{4 2}$ $167-179$

Morrell JM and Dresser DW (1989) Offspring from inseminations with mammalian sperm stained with Hoechst 33342, either with or without flow cytometry Mutation Research 224 177-183

Morrell JM, Keeler KD, Noakes DE, Mackenzie NM and Dresser DW (1988) Sexing of sperm by flow cytometry Veterinary Record 122 322-324

Moss JA, Melrose DR, Reed HCB and Vandeplassche M (1979) Fertility and Infertility in Domestic Animals. 3rd edn, Ed. JA Laing. Bailliere Tindall, London

Napier R (1963) Animals in Research: Principles of Breeding and Management p 345 Ed W Lane-Petter. Academic Press, London

Shapiro HM (1988) Practical Flow Cytometry. Second edition, Liss, New York

Stovel RT, Sweet RG and Herzenbeg LA (1978) A means for orientating flat cells in flow systems Biophysical Joumal 23 1-5

Van Dilla MA, Gledhill BL, Lake S, Dean PN, Gray JW, Kachel V, Barlogie B and Gohde W (1977) Measurement of mammalian sperm deoxyribonucleic acid by flow cytometry. Problems and approaches Journal of Histochemistry and Cytochemistry 25 763-773 